\title{
PREDITORES DE RETENÇÃO E LEALDADE DE CLIENTES EM ACADEMIAS DE GINÁSTICA ${ }^{1}$
}

\section{PREDICTORS OF RETENTION AND CUSTOMER LOYALTY IN GYMS}

\author{
David de França Arcoverde* E-mail: dafarc@hotmail.com \\ Marcus Augusto Vasconcelos Araújo*E-mail: marcusaugusto77@hotmail.com \\ ${ }^{*}$ Faculdade Boa Viagem-DeVry (DeVry FBV), Recife, PE
}

\begin{abstract}
Resumo: A lealdade dos clientes deve ser encarada como uma vantagem sustentável e indispensável à sobrevivência de uma organização de serviços. Por isso, acadêmicos e profissionais têm se engajado nos últimos anos em estratégias voltadas para o aumento na percepção da qualidade do serviço e de barreiras que dificultem o abando precoce dos serviços contratados. O objetivo deste estudo foi verificar a influência da qualidade percebida e das barreiras relacionais à mudança na lealdade e na retenção de clientes em academias de ginástica. A pesquisa, de natureza descritiva, foi realizada em duas academias de ginástica com uma amostra $n=228$. As dimensões de Qualidade e de Barreiras Relacionais à Mudança foram tomadas como variáveis independentes e submetidas à análise de regressão logística para retenção e para lealdade. Os resultados demonstraram que os aspectos tangíveis associados ao serviço são a dimensão da qualidade que mais afeta a lealdade. $\mathrm{O}$ estudo preditivo da retenção apresentou a frequência de utilização do serviço como sendo a variável mais importante associada ao comportamento de recompra, seguido de empatia. Conclui-se que a qualidade de serviço e as barreiras influenciam positivamente na lealdade e na retenção de clientes, embora existam preditores diferentes para a retenção e a lealdade. Além disso, a qualidade tem um maior poder de influência do que as barreiras relacionais, especialmente na lealdade.
\end{abstract}

Palavras-Chave: Lealdade. Retenção. Qualidade de Serviço. Barreiras à Mudança. Academia de Ginástica.

\begin{abstract}
Customer loyalty must be viewed as a sustainable and indispensable advantage to the survival of a service organization. For this reason, academics and professionals have been engaged in working on strategies which aim at increasing the perception of service quality and barriers that prevent desertion. The objective of this study was to verify the influence of the perceived quality and the relational barriers to the change in customer loyalty and retention in gyms. The research, which has descriptive nature, was performed in two gymnasiums with a sample $n=228$. The dimensions of Quality and Relational Barriers to Change were taken as independent variables and submitted to logistic regression analysis for retention and for loyalty. The results demonstrated that the tangible aspects associated with service are the dimension of quality that most affects loyalty. The predictive study of retention showed the frequency of service utilization as the most important variable associated with repurchasing, followed by empathy. We conclude that quality of service and barriers positively influence loyalty and customer retention although there are different predictors of retention and loyalty. In addition, quality has a greater power of influence than relational barriers, especially regarding loyalty.
\end{abstract}

Keywords: Loyalty. Retention. Service Quality. Barriers to Change. Gyms.

\footnotetext{
${ }^{1}$ Artigo apresentado originalmente como dissertação de mestrado.
} 


\section{INTRODUÇÃO}

O fim do monopólio comercial e a livre concorrência, aliados ao acesso cada vez mais rápido à informação e equiparação tecnológica têm dado aos consumidores a oportunidade de escolher entre empresas concorrentes, uma vez que essas empresas compartilham o potencial de entregar um mesmo produto (VAVRA, 1999; DRUCKER, 2002; PORTER, 2004). Isso favoreceu uma crescente busca por diferenciação e vantagem por parte das empresas, a fim de alcançarem um número cada vez maior de clientes. Vavra (1999, p.26) denominou "marketing de conquista" como sendo o esforço das organizações para conquistar novos clientes. Tal esforço, no entanto, negligenciava os clientes atuais, como se eles fossem uma aquisição permanente.

Este ambiente econômico se tornou um grande obstáculo para o atingimento da fidelidade. Os consumidores têm sido atacados por uma "multiplicidade de alternativas aparentemente superiores" de produtos e serviços, que terminam por modificar de forma acelerada suas expectativas em relação a produtos e serviços (OLIVER, 1999, p.37). Esse cenário é extremamente complexo e dinâmico e implica numa visão cada vez mais relacional e menos transacional para as trocas de valor entre cliente e fornecedor, levando ao surgimento do marketing de relacionamento (MORGAN; HUNT, 1994; LOVELOCK; WRIGHT; HEMZO, 2011). O foco deixa de ser exclusivamente a aquisição de novos clientes e passa a ser, concomitantemente, a manutenção dos atuais.

Neste contexto, a lealdade dos clientes deve ser encarada pelas organizações como uma vantagem sustentável (DICK; BASU, 1994). Reichheld (2001, p. 9) afirma que "muito provavelmente, a única fonte possível de vantagem competitiva sustentável na nova economia serão os laços de lealdade que uma empresa for capaz de gerar com seus clientes".

Um argumento comum a favor da fidelização de clientes consiste no fato de que conquistar novos clientes custa mais caro para a empresa do que manter os clientes atuais (VAVRA, 1993; MORGAN; HUNT, 1994; OLIVER, 1999; PAYNE, 2006). Neste mesmo sentido, para demonstrar a importância de manter relacionamentos duradouros com os clientes, Reichheld (2001, p.10) afirma que "um acréscimo de $5 \%$ na taxa de retenção pode aumentar os lucros entre $25 \%$ e $90 \%$ ". O autor acrescenta 
que os clientes são mais lucrativos a depender do tempo de relacionamento com a empresa. Dessa forma, quanto maior o tempo de relacionamento, mais o cliente comprará e menos demandará da empresa em termos de suporte.

Entretanto, fortalecer laços de lealdade com os clientes não é tarefa fácil, uma vez que essa relação com o consumidor sofre influência de diversos fatores, entre eles: a satisfação dos clientes, a qualidade percebida do serviço, o valor percebido e as barreiras à mudança (FORNELL et al., 1996; PAN; SHENG; XIE, 2012).

Sendo assim, diversos construtos e características de produtos e serviços têm sido estudadas, levando ao desenvolvimento de métricas sofisticadas para o gerenciamento das expectativas e da percepção dos clientes (GUPTA; ZEITHAML, 2006). Concomitantemente, o registro amplo de dados dos clientes tem aberto as portas ao aprimoramento de modelos e métodos preditivos para o gerenciamento da rotatividade de clientes e da lucratividade das empresas (NESLIN; GUPTA; KAMAKURA; MASON, 2006).

O mercado do fitness tem se beneficiado com o marketing de relacionamento, especialmente pelo uso das ferramentas de $\mathrm{CRM}^{2}$. As academias de ginástica têm utilizado cada vez mais os softwares de gerenciamento e bancos de dados em suas rotinas, a fim de tornar eficaz e lucrativo seu relacionamento com os clientes, em um compromisso claro com a profissionalização do setor (ENTRIEL, 2008; ARAÚJO, 2010; PORTO; SILVA, 2013).

Entretanto, estudos têm demonstrado que 50\% dos alunos de academias deixam de frequentá-las nos primeiros 6 meses (ROJAS, 2003; ARAÚJO, 2010) e apenas $64,4 \%$ são retidos e efetuam uma nova compra (MCCARTHY, 2004). Isso pode apontar para o fato de que as informações atualmente monitorizadas têm pouca relevância; o tratamento das informações dos clientes é insuficiente ou não se está recebendo o suporte organizacional adequado para transformar essas informações em resultados de retenção (RIGBY, 2004).

O atual modelo de negócio precisa ser repensado. As academias precisam assumir um compromisso mais profundo com a qualidade de seu serviço e a satisfação dos clientes, a fim de atingir a lealdade de seus consumidores e crescer de

\footnotetext{
${ }^{2}$ Gestão de relacionamento com o cliente.
} 
forma sustentável. Os gestores, entretanto, parecem desconsiderar, em sua rotina de gerenciamento, importantes preditores da lealdade (PAN; SHENG; XIE, 2012).

A partir dessas considerações, apresenta-se a seguinte questão de pesquisa: Qual a influência da qualidade percebida e das barreiras relacionais sobre a mudança na lealdade e no comportamento de recompra em academias de ginástica?

\subsection{Objetivos}

Geral

Verificar a influência da qualidade percebida e das barreiras relacionais sobre a mudança na lealdade e no comportamento de recompra em academias de ginástica.

\section{Específicos}

- Verificar a relação entre lealdade e retenção de clientes em academias de ginástica;

- Verificar qual a relação da qualidade percebida e barreiras relacionais à mudança com a lealdade de clientes em academias de ginástica;

- Verificar qual a relação da qualidade percebida e barreiras relacionais à mudança com a retenção de clientes em academias de ginástica;

- Verificar qual a relação entre as características individuais dos clientes e a retenção.

\subsection{Justificativa}

Esse trabalho é relevante para a área do Marketing de Relacionamento no setor de serviços, especialmente por trazer num mesmo modelo uma medida de lealdade atitudinal e o comportamento de compra/renovação do serviço. Em sua maioria, os demais trabalhos estabelecem como variável dependente apenas a lealdade, e esta, na perspectiva da intenção comportamental (CRONIN; TAYLOR; 1992; TAYLOR; BAKER, 1994; CRONIN; BRADY; HULT, 2000).

Pesquisar os antecedentes da retenção e da lealdade é uma opção que se baseia na proposição de que os preditores da lealdade e da retenção não são necessariamente os mesmos, o que deveria provocar um esforço para se elucidar os 
benefícios e peculiaridades de cada um. Esta pesquisa pode, portanto, impactar diretamente nas estratégias de marketing de relacionamento das organizações, através do estabelecimento mais consciente de seus objetivos, a saber: aumentar a taxa de indivíduos leais, aumentar a taxa de retenção ou ambos. Além disso, poucas pesquisas têm incluído a "recompra" em seu conjunto de variáveis, sendo essa a medida de comportamento do cliente que, em última instância, mais interessa ao mercado do fitness (CHELLADURAI; CHANG, 2000).

\section{DESENVOLVIMENTO}

Manter relacionamentos duradouros entre fornecedor e cliente é função do marketing de relacionamento. Morgan e Hunt (1994, p.22) propõem que o "Marketing de Relacionamento se refere a todas as atividades de marketing voltadas para o estabelecimento, desenvolvimento e manutenção de trocas relacionais bemsucedidas". Está ancorado na premissa básica de que é entre 5 e 6 vezes mais caro conquistar um novo cliente do que implementar estratégias para manter os clientes atuais (LOVELOCK; WRIGHT; HEMZO, 2011).

A seguir, serão apresentados os construtos retenção e lealdade; suas diferenças conceituais, assim como seus antecedentes: qualidade e barreiras à mudança.

\subsection{Retenção (recompra)}

Retenção é essencialmente o comportamento de repetição de uma compra, e a medida da retenção resulta num índice geral da probabilidade de permanência ou recompra de serviço (GUMTA; ZEITHAML, 2006). Nota-se que a variável retenção independe de o cliente estar ou não satisfeito com o serviço (OLIVER, 1999), ela apenas o observa.

É importante distinguir entre dois aspectos da retenção: o primeiro é positivo e coincide com o comportamento de lealdade. Não deve ser confundido, no entanto, com a intenção comportamental (OLIVER, 1999). O segundo é negativo e reflete uma falsa lealdade (DICK; BASU, 1994; WHITE; YANAMANDRAN, 2004), que será melhor compreendida na próxima seção. A falsa lealdade, embora pareça boa, não produz resultados significativos a longo prazo. Reichheld (2012) alerta para a lucratividade 
negativa, proveniente de clientes retidos apenas pelas barreiras de mudança ou indiferença. Esses clientes são um risco iminente, à medida em que podem facilmente trocar a prestadora do serviço por uma concorrente. Então, se a maior parte dos clientes de uma empresa apresenta baixos índices de lealdade, tal empresa está correndo sérios riscos.

A retenção de clientes não acontece de maneira uniforme para todos os segmentos de clientes. Os estudos têm apontado respostas diferentes em cada segmento à probabilidade de recompra no contexto de serviços (PATTERSON; 2007) inclusive em se tratando do mercado de fitness (AFTHINOS; THEODORAKIS; NASSIS, 2005; GARCÍA; SAÑUDO, 2013).

Um dos aspectos de segmentação mais relevantes é o perfil de utilização do serviço, medido pela frequência e pelo tempo de permanência na academia. As pessoas que frequentam a academia com maior regularidade ou permanecem mais tempo por dia, têm maior aderência aos programas de atividades (ROJAS, 2003; SOUZA et al., 2009; PORTO; SILVA, 2013; MCCARTHY, 2004).

Sendo assim, apenas a venda dos pacotes não é garantia da fidelização de clientes. É necessário que o cliente usufrua do serviço. Contudo, no caso de não haver frequência regular, corre-se o risco de que aquele não perceba o valor do serviço por ele contratado (MCCARTHY, 2004). Souza et al. (2009) afirmam que a retenção do cliente é consequência do aumento na utilização de produtos por parte do cliente.

O gerenciamento da frequência de acessos é de vital importância, uma vez que este dado refere-se diretamente à utilização do serviço. Se o cliente está pagando por algo que não está sendo utilizado, é natural que ele perceba como desperdício o valor pago pela mensalidade. Aderência está diretamente relacionada com a retenção (ARAÚJO, 2010), embora seja apenas um dos fatores que se associam com a não efetivação de uma nova contratação. Sabá (2001, p.61) define aderência como o "comprometimento dos praticantes de exercício físico com a rotina programada de treinamento."

\subsection{Lealdade}

Lealdade, enquanto constructo, tem sido abordada na literatura a partir de três dimensões conceituais (FERRAND et al., 2010; YOO; BAI, 2013; ROCHA, 2013). 
Primeiro, comportamental, onde lealdade implica recompra ou renovação. Segundo, atitudinal, envolvendo uma atitude favorável a determinado produto ou serviço. A terceira dimensão é a mais aceita, sendo ela a combinação dos componentes atitudinal e comportamental.

Lealdade é uma relação que se estabelece em diversas situações, como entre cliente e fornecedor, empregado e empregador, entre casais, amigos, etc. Lealdade do cliente, segundo Reichheld (2003, p.3), significa "permanecer com um fornecedor que o trata bem e lhe dá um bom valor no longo prazo, mesmo que o fornecedor não ofereça o melhor preço em uma transação particular." Já para Oliver (1999, p.34), lealdade é:

\begin{abstract}
Um profundo compromisso para a recompra ou renovação de um produto/serviço preferido de forma consistente no futuro, causando assim, compras repetitivas da mesma marca ou algum conjunto de marcas, apesar das influências situacionais e dos esforços de marketing com o potencial de causar o comportamento de mudança.
\end{abstract}

A lealdade, evidentemente, influencia na retenção de clientes. Há de se considerar, no entanto, que nem sempre o comportamento de recompra reflete a lealdade de um cliente em relação a uma marca, conforme proposto por Dick e Basu (1994). Por exemplo, um indivíduo pode se manter cliente de uma determinada empresa "por inércia, indiferença, por uma barreira à mudança ou por determinada circunstância" (REICHHELD, 2003 p.3) sem, contudo, evidenciar nenhum grau de favorabilidade ou preferência à mesma, o que indica um quadro de "falsa lealdade". Por outro lado, uma pessoa pode estar momentaneamente impossibilitada de frequentar uma academia devido a alguma lesão e, mesmo assim, continuar fazendo comunicação positiva para amigos e familiares e incentivando outras pessoas a procurarem a mesma, determinando um quadro de "lealdade latente". É relevante destacar que normas sociais, aspectos situacionais e percepção de controle, entre outros fatores, são reconhecidos na literatura como capazes de modificar a relação entre a atitude e o comportamento de lealdade (AJZEN, 1991).

Dick e Basu (1994) utilizaram o termo "atitude relativa" para descrever o grau de favorabilidade e preferência a uma marca frente à existência de outros potenciais fornecedores. Entretanto, para esses autores, a lealdade verdadeira apenas seria observada quando houvesse a coincidência de uma atitude relativa alta e o comportamento de recompra, numa perspectiva composta para a lealdade (atitude e 
comportamento). A Figura 1 demonstra que, embora possam se manifestar distintamente, existe um ponto comum entre a lealdade e retenção.

Na prática, essa perspectiva atitudinal e comportamental da lealdade não tem sido aplicada em pesquisas acadêmicas, o que poderia demandar uma ampla modificação nas metodologias utilizadas. Por outro lado, Yue Pan, Simon Sheng e Frank T. Xie (2012) afirmam que a escolha dos instrumentos para a medição da lealdade tem sido arbitrária, o que também é descrito por Gupta e Zeithaml (2006).

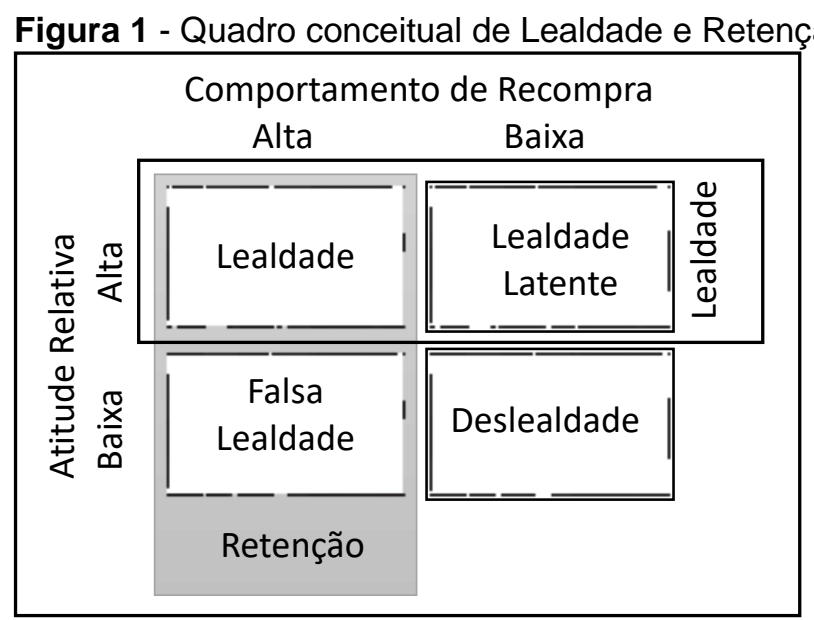

Fonte: Dick e Basu (1994, p.101), adaptado pelo autor

Embora se afirme que as medidas de atitude sejam boas substitutas para as medidas comportamentais, isto não tem sido testado (PAN et al., 2012). Nesta pesquisa, existe a intenção de o serviço como medida suficiente para a determinação da lealdade (REICHHELD, 2003), partindo do pressuposto de que ela compreende a dimensão afetiva/atitudinal da lealdade (OLIVER, 1999; DICK; BASU, 1994). Uma consideração importante a respeito da intenção de recomendar é que ela, por si só, já é uma atitude desejável por conta do poder que as recomendações positivas têm de influenciar na decisão de compra de outros consumidores (BUTTLE, 1998).

A partir do que foi exposto até o momento, chega-se à seguinte hipótese: H1: A Lealdade dos clientes se relaciona direta e positivamente com a retenção de clientes. 


\subsection{Qualidade em Serviços}

No contexto de serviços, a palavra qualidade pode ser empregada a partir de várias perspectivas: eficiência operacional, maximização do valor, como algo inato (transcendente), ou ainda, como a percepção de excelência do serviço por parte do cliente (LOVELOCK; WIRTZ; HEMZO, 2011; RUST; MOORMAN; DICKSON, 2002).

$\mathrm{Na}$ perspectiva dos clientes, diversas estratégias têm sido utilizadas para o gerenciamento da qualidade a exemplo do método Desdobramento da Função Qualidade (QFD). Este método identifica lacunas entre as expectativas e do desempenho dos serviços, e contribui para a definição de requisitos técnicos para o serviço. Este método, tem o objetivo de transformar as necessidades dos clientes em requisitos técnicos a fim de maximizar a percepção de excelência do serviço. Muito embora, pesquisas recentes tenham se voltado mais para a monitorização apenas das expectativas dos clientes e menos para o desempenho de seus serviços (DE SOUZA; MIGUEL, 2017).

Neste sentido, para compreender a qualidade é necessário entender como os clientes percebem e avaliam a qualidade. Parasuraman, Zeithaml e Berry $(1985,1988)$ e PBZ $^{3}$ (1991) identificaram cinco dimensões, a partir das quais, usuários seriam capazes de avaliar a qualidade do serviço:

- Tangibilidade - Aparência das instalações físicas, equipamentos e pessoal;

- Confiabilidade (credibilidade) - Capacidade de executar o serviço prometido de forma confiável e com precisão;

- Presteza (responsividade) - Vontade de ajudar os clientes e fornecer um pronto atendimento;

- Segurança - Conhecimento e cortesia dos funcionários, e sua habilidade para inspirar confiança e segurança;

- Empatia - Carinho e atenção individualizada que a empresa fornece aos seus clientes.

A partir da constatação dessas dimensões, o consumidor forma uma expectativa e um juízo geral da excelência do serviço, que é denominado qualidade

\footnotetext{
${ }^{3}$ São os mesmos autores citados, porém numa ordem diferente.
} 
percebida, e difere da satisfação do cliente, uma vez que esta é mais apropriada para descrever uma reação emocional posterior a uma transação específica (PARASURAMAN; ZEITHAML; BERRY, 1988).

Qualidade de serviços tem sido citada na literatura como um antecedente direto da lealdade (TAYLOR; BAKER, 1994), indireto (FORREL et al., 1996) e ambos (CRONIN et al., 2000), sendo comum a todos os modelos, algum nível de relação com a satisfação dos clientes. A maioria das medidas, entretanto, compreendem a intenção comportamental de recomprar o serviço, e não propriamente a retenção dos clientes (CRONIN et al., 2000).

Com o fim de estabelecer uma métrica para a quantificação da qualidade percebida por parte dos consumidores, Parasuraman, Zeithaml e Berry $(1985,1988)$ e PBZ (1991) desenvolveram a escala SERVQUAL. Trata-se de um instrumento contendo 22 perguntas, compreendidas nas 5 dimensões supracitadas. Cronin e Taylor (1992, 1994) propuseram uma simplificação do SERVQUAL (SERVPERF), reduzindo assim a um terço, o tamanho do instrumento de coleta, simplificando a coleta e análise dos dados.

Outra medida importante na mensuração da qualidade são as questões de ambiente de serviço, ou Servicescape. Com relação a esta, é importante destacar que uma das características distintivas do Marketing de Serviços é a compreensão de que a produção e o consumo acontecem de forma simultânea (GRÖNROOS, 1982). Assim, o ambiente no qual se materializa a transação de serviço, é também sua "fábrica". Esse ambiente influencia a percepção da qualidade do serviço, na medida em que não é possível dissociar o serviço em si do ambiente de sua concepção (BITNER, 1992). Essa visão integrada é reforçada por Hoffman e Turley (2002). Para os autores, o ambiente em que o encontro de serviço acontece, fornece "evidências" que ajudam os clientes a avaliarem o serviço. Nesta pesquisa, os aspectos ambientais contemplados serão: música, temperatura e o layout dos equipamentos (disposição espacial).

Com base no que foi exposto nesta seção, propõem-se as seguintes hipóteses: H2: A Qualidade Percebida influencia direta e positivamente a Lealdade de Clientes. H3: A Qualidade Percebida influencia direta e positivamente a Retenção de Clientes. 


\subsection{Barreiras à Mudança}

Embora a melhor maneira de fortalecer a retenção esteja relacionada com a entrega de um serviço que proporcione um alto grau de satisfação para os clientes, outro método, também importante, consiste em "[...] erguer barreiras elevadas para impedir a mudança." (KOTLER; KELLER, 2006, p.153).

Jones, Mothersbaugh e Beatty (2000), definem Barreiras à Mudança como sendo "todos os fatores que tornam mais difícil ou custoso, para os consumidores, mudar de fornecedor" (p. 261). Para esses autores, Barreiras à Mudança englobam:

- A Percepção dos Custos de Mudança;

- Os Relacionamentos Interpessoais (benefícios relacionais); e

- A falta de atratividade dos fornecedores alternativos.

Custos de Mudança são, de uma forma geral, os fatores que limitam ou dificultam o processo de mudança de um fornecedor para outro, envolvendo expectativas de perda e riscos. (MORGAN; HUNT, 1994). Ou, simplesmente, os custos, não contínuos, que os clientes associam ao processo de mudança (BURNHAM et al., 2003).

Para esta pesquisa foi adotada a tipologia de Burnham, Frels e Mahajan (2003), porém, apenas os custos de perda de relacionamento pessoal serão investigados. Esta restrição se justifica pela identificação da importância que os relacionamentos têm, em especial, para o tipo de serviço pesquisado.

Benefícios sociais são os benefícios relacionais que envolvem a familiaridade, reconhecimento pessoal, amizade, harmonia e suporte social. Este tipo de benefício é ainda mais importante em serviços nos quais existe maior contato com o cliente, como serviços médicos, salão de beleza e serviços de fitness (GWINNER; GREMLER; BITNER, 1998; PATTERSON; SMITH, 2003).

A partir do que foi exposto nesta seção, propõem-se as seguintes hipóteses: H4: As Barreiras Relacionais à Mudança se relacionam direta e positivamente com a Lealdade dos clientes.

H5: As Barreiras Relacionais à Mudança se relacionam direta e positivamente com a Retenção de Clientes. 


\subsection{Modelo Conceitual e Hipóteses}

A partir do que foi visto na literatura, foram propostas 05 hipóteses relacionando os principais temas desenvolvidos. A Figura 2 representa graficamente o modelo hipotético que será testado.

Figura 1 - Modelo conceitual



Fonte: Elaborado pelo autor

\section{METODOLOGIA}

Esta pesquisa tem natureza descritiva, pois se apoia na observação, acompanhamento e descrição de características de uma determinada população (PRODANOV; FREITAS, 2013, p.52). É, ainda, quantitativa e, quanto ao tempo, retrospectiva, uma vez que algumas das variáveis utilizadas neste trabalho refletem comportamentos no passado.

A população desta pesquisa consta de frequentadores de academias de ginástica da cidade de Recife-PE. Quanto à amostra, para a operacionalização da pesquisa, foram utilizadas duas academias da cidade: uma localizada no bairro da Torre, na Zona Norte, com 1253 alunos cadastrados, sendo 707 ativos. Outra no bairro da Estância, Zona Oeste do Recife, com 1927 alunos cadastrados, sendo 783 ativos. O método de amostragem adotado foi o de amostra por conveniência. 
Com relação à coleta de dados, dois métodos foram utilizados para obtenção de informações na população pesquisada:

- Método de Levantamento de Campo (survey) - Foi aplicado um questionário contendo 33 questões, incluindo as 22 questões da SERVQUAL, 03 questões sobre os fatores/aspectos ambientais, 07 questões sobre barreiras relacionais à mudança e 01 questão sobre lealdade.

- Método de Observação Mecânica Indireta - Este método se utiliza de dispositivos mecânicos (neste caso, uma catraca) para registrar o comportamento de indivíduos, não modificando, contudo, sua rotina (MALHOTRA, 2006). Este método de observação foi utilizado na coleta de informações a respeito da frequência de acessos semanais à academia.

Além dos dados obtidos pela coleta de dados, as informações do cadastro de alunos disponíveis no sistema de controle da academia, também foram utilizados nas análises.

A quantidade final de questionários válidos foi de 228, sendo 122 respondentes do sexo feminino e 106 do sexo masculino, representando respectivamente $53,5 \%$ e $46,5 \%$. A idade média dos respondentes é de 33,3 anos com um desvio padrão de 12,3 , sendo as idades mínima e máxima, respectivamente, 14,9 e 77,4 anos. Jovens entre 21 e 30 anos integram o grupo que mais respondeu a pesquisa.

Técnicas de análise:

- Análise Fatorial Confirmatória (AFC) - Neste trabalho ela será utilizada com o propósito de validar o instrumento de mensuração. É uma técnica multivariada que tem como objetivo "testar o quão bem variáveis medidas representam um número menor de construtos" (HAIR et al., 2006, p. 589).

- Análise de correlação de Spearman - Esta técnica foi utilizada para a análise da relação entre as variáveis 'retenção' e 'lealdade'

- Análise de Regressão Logística - Esta técnica é um tipo de análise de regressão múltipla e tem por objetivo usar as variáveis independentes com valores conhecidos para prever os valores de uma variável dependente binária. As variáveis dependentes deste estudo foram lealdade e retenção; sendo assim, foram geradas duas análises distintas. 
As análises foram realizadas com o uso do software Statistical Package for the Social Sciences (SPSS) versão 22 e o complemento Amos, especificamente para a Análise Fatorial Confirmatória.

\section{RESULTADOS}

\subsection{Instrumento}

A redução dos itens do questionário nas dimensões propostas pela teoria foi submetida à validação por meio da Análise Fatorial Confirmatória, através da estimação de máxima verossimilhança. Os valores obtidos para RMSEA (root mean square error of approximation) e SRMR (standardised root mean square residual) foram respectivamente 0,073 e 0,0644, e o índice de ajuste incremental CFI foi de 0,90, retornando um resultado satisfatório (HAIR et al. 2009).

A confiabilidade do instrumento também foi testada e os valores alfa $(\alpha)$ de Cronbach variaram entre 0,722 e 0,874 .

\subsection{Correlação entre Lealdade e Retenção}

O teste de correlação de Spearman foi utilizado para a análise da relação entre as variáveis 'retenção' e 'lealdade'. Este teste é o "equivalente não paramétrico para o coeficiente de correlação de Pearson" (LARSON; FARBER, 2010, p. 508), podendo ser utilizado para dados não contínuos, como é o caso da variável dicotômica lealdade. Esta etapa corresponde ao primeiro objetivo específico proposto para esta pesquisa e a primeira hipótese. O resultado foi de 0,150 , significativo no nível de confiança de 0,05 , indicando uma correlação positiva.

A hipótese $\mathrm{H} 1$ não foi refutada. A relação entre lealdade e retenção existe, embora esta relação seja fraca.

\subsection{Estudo preditivo}

A análise de regressão logística foi a técnica estatística utilizada nesse estudo por sua particularidade em aceitar uma medida binária para a variável dependente 
(HAIR et al., 2009). Dois testes foram realizados, sendo um para cada uma das variáveis dependentes, 'retenção' e 'lealdade'. O quadro 1 descreve cada uma das variáveis pesquisadas nos modelos.

Quadro 1 - Descrição das variáveis utilizadas na regressão

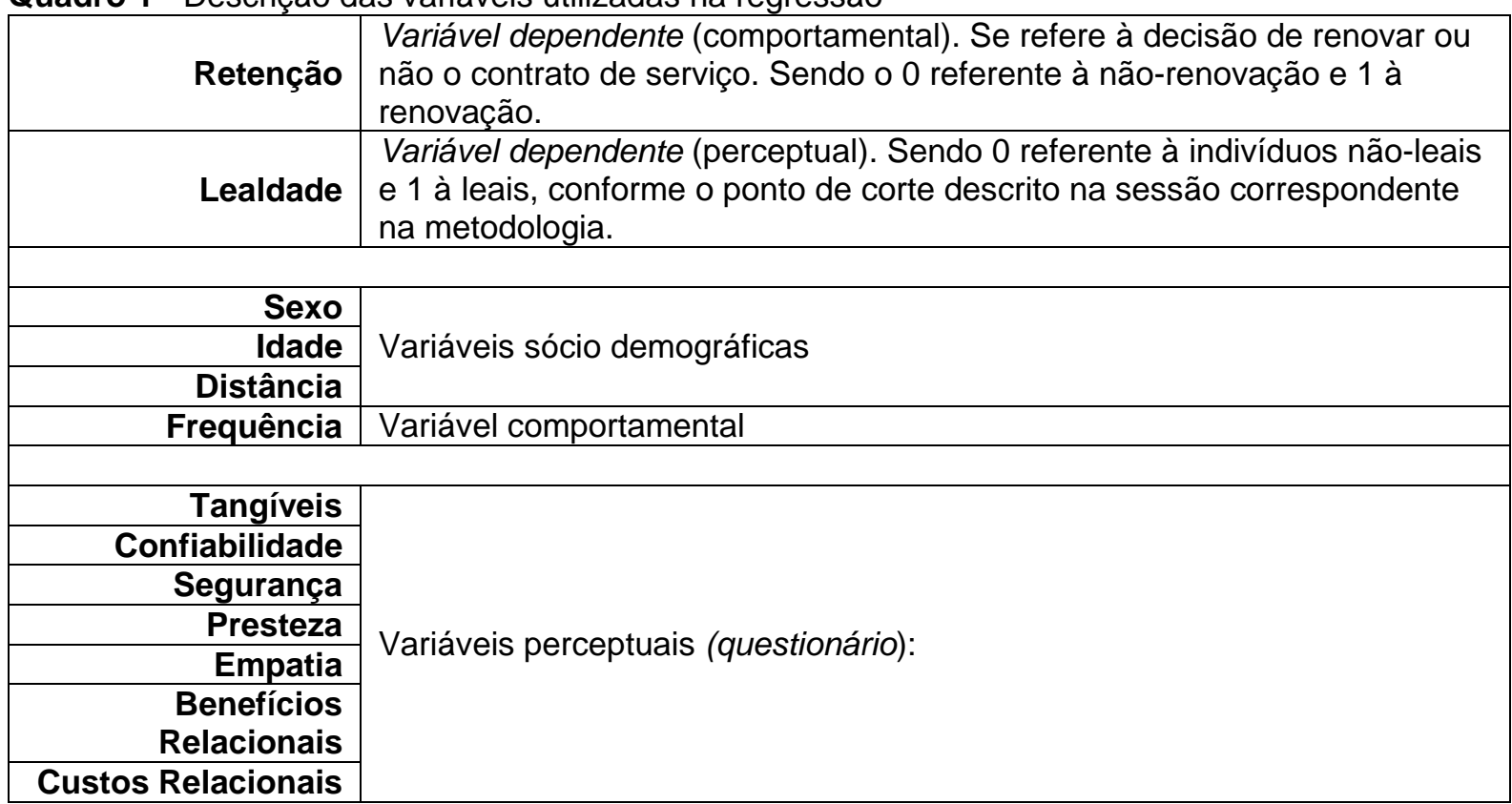

Fonte: Elaborado pelo autor

O método empregado foi o Forward LR (Likelihood Ratio), que é um método de seleção por etapas que leva em consideração a significância da estatística de score para inclusão de variáveis (SPSS, 2015).

Primeiro foram feitos os testes para a variável Lealdade:

Quadro 2 - Quadro descritivo da estimação para Lealdade

\begin{tabular}{|r|l|l|l|}
\hline \multirow{2}{*}{} & \multicolumn{3}{|c|}{ Porcentagem global de acertos (apenas a constante) } \\
\cline { 2 - 4 } & \multicolumn{3}{|c|}{ Porcentagem global de acertos (constante + variáveis) } \\
\cline { 2 - 4 } & \multicolumn{3}{|c|}{$77,6 \%-3$ etapas } \\
\cline { 2 - 4 } & Verossimilhança de log-2 & R quadrado Cox \& Snell & R quadrado Nagelkerke \\
\hline inicial & 240,120 &, 204 &, 280 \\
\hline final & 214,935 &, 289 &, 397 \\
\hline \multirow{3}{*}{ Tangíveis; Segurança; Custos Relacionais } \\
\cline { 2 - 4 } & \multicolumn{3}{|c|}{ Variáveis aceitas } \\
\hline
\end{tabular}

Fonte: Elaborado pelo autor 
A redução nos valores da -2 logaritmo de verossimilhança (-2LL) indicam um melhor ajuste no modelo de regressão (HAIR et al., 2009). O teste obteve significância estatística no nível 0,000 .

As medidas de pseudo $\mathrm{R}^{2}$ Cox \& Snell e Nagelkerke obtiveram aumento considerável, conforme apresentado no Quadro 2, e a percentagem global de acertos é superior no modelo com as variáveis quando comparado ao modelo apenas com a constante.

Por fim, a tabela a seguir apresenta os valores do coeficiente logístico exponenciado (odds ratio) para cada variável na equação. Esses valores menos um, representam a variação percentual da razão de desigualdade. Sendo assim, cada variação positiva de um ponto para as variáveis Tangíveis e Segurança aumentam respectivamente em 3,9 e 2,4 vezes a chance para a variável dependente.

Tabela 1 - Variáveis aceitas (lealdade)

\begin{tabular}{|c|c|c|c|c|c|c|}
\hline & B & S.E. & Wald & df & Sig. & $\operatorname{Exp}(B)$ \\
\hline Tangíveis & 1,592 & ,372 & 18,271 & 1 & ,000 & 4,912 \\
\hline Segurança & 1,232 & ,376 & 10,706 & 1 & ,001 & 3,427 \\
\hline $\begin{array}{l}\text { Custos } \\
\text { Relacionais }\end{array}$ & ,484 & ,159 & 9,238 & 1 & ,002 & 1,622 \\
\hline Constante & $-12,413$ & 1,974 & 39,560 & 1 & ,000 &, 000 \\
\hline
\end{tabular}

Fonte: Elaborado pelo autor

No modelo preditivo da lealdade, a relação mais forte encontrada foi relacionada com os aspectos tangíveis. Esta dimensão esteve presente no modelo gerado para a lealdade num nível de significância de 0,01 e com um alto valor odds ratio. Isso leva a crer que, para o setor de academias de ginástica, uma boa infraestrutura, organização, modernidade de equipamentos e de recursos, agradabilidade de música e temperatura são indispensáveis para o atingimento da lealdade dos clientes. Este resultado está em consonância com os achados de Afthinos, Theodorakis e Nassis (2005), autores que estudaram as expectativas de clientes em Academias privadas e públicas. Nas academias privadas, 'modernidade dos equipamentos' era uma das maiores expectativas dos clientes. Segurança também foi uma dimensão da qualidade que influenciou na lealdade.

A hipótese $\mathrm{H} 2$ foi parcialmente aceita, uma vez que duas dimensões da qualidade propostas na teoria foram contempladas nos modelos, com destaque para os aspectos tangíveis. 
A hipótese $\mathrm{H} 4$ foi parcialmente aceita. Os 'custos relacionais' foram aceitos e 'benefícios sociais' rejeitados.

Pode-se interpretar esses resultados da seguinte forma: nem sempre a existência de vínculos de relacionamento no ambiente de serviço será suficiente para tornar os indivíduos mais leais. É necessário que os clientes percebam como um custo a perda deste relacionamento.

Em seguida foram feitas as estimações para a variável Retenção.

Quadro 3 - Quadro descritivo da estimação para Retenção

\begin{tabular}{|l|l|l|l|}
\hline \multirow{2}{*}{} & \multicolumn{3}{|c|}{ Porcentagem global de acertos (apenas a constante) } \\
\cline { 2 - 4 } & \multicolumn{3}{|c|}{$81,7 \%$} \\
\cline { 2 - 4 } & \multicolumn{3}{|c|}{ Porcentagem global de acertos (constante + variáveis) } \\
\cline { 2 - 4 } & Verossimilhança de log-2 & R quadrado Cox \& Snell & R quadrado Nagelkerke \\
\hline inicial & 75,748 &, 295 &, 481 \\
\hline final & 70,790 &, 322 &, 525 \\
\hline \multirow{3}{*}{} & \multicolumn{3}{|c|}{ Variáveis aceitas } \\
\cline { 2 - 4 } & \multicolumn{3}{|c|}{ Frequência; Empatia (confi5) } \\
\hline
\end{tabular}

Fonte: Elaborado pelo autor

A análise apresentou redução nos valores da -2 logaritmo de verossimilhança (-2LL) indicando que a inclusão das variáveis independentes melhorou o ajuste do modelo. Houve significância estatística no nível 0,000.

As medidas de pseudo $\mathrm{R}^{2}$ Cox \& Snell e Nagelkerke obtiveram aumento considerável nas duas medidas, conforme apresentado no Quadro , e a percentagem global de acertos é superior no modelo com as variáveis quando comparado ao modelo apenas com a constante.

Os valores do coeficiente logístico exponenciado (odds ratio) para cada variável na equação são apresentados no quadro a seguir. Considerando que esses valores menos um, representam a variação percentual da razão de desigualdade, cada variação positiva de um ponto para a variável Empatia aumenta em 1,7 vezes a chance para a variável dependente no modelo. 
Tabela 2 - Variáveis aceitas (retenção)

\begin{tabular}{|c|r|r|r|r|r|r|}
\hline & \multicolumn{1}{|c|}{ B } & \multicolumn{1}{|c|}{ S.E. } & \multicolumn{1}{|c|}{ Wald } & \multicolumn{1}{|c|}{ df } & \multicolumn{1}{c|}{ Sig. } & \multicolumn{1}{|c|}{$\operatorname{Exp(B)}$} \\
\hline Frequência & 1,955 &, 430 & 20,629 & 1 &, 000 & 7,062 \\
\hline Empatia & 1,024 &, 477 & 4,608 & 1 &, 032 & 2,785 \\
\hline Constante & $-5,341$ & 2,121 & 6,340 & 1 &, 012 &, 005 \\
\hline
\end{tabular}

Fonte: Elaborado pelo autor

A hipótese H3 foi parcialmente aceita. A dimensão Empatia apresentou um valor de odds ratio alto no modelo 3 .

A hipótese H5 não foi aceita, uma vez que nenhuma das Barreiras à Mudança aqui estudadas foram relacionadas com a 'retenção'. Isto não descarta a possibilidade de que outras Barreiras poderiam apresentar resultados diferentes, caso fossem incluídas neste trabalho.

Quadro 4 - Quadro descritivo da estimação para Retenção

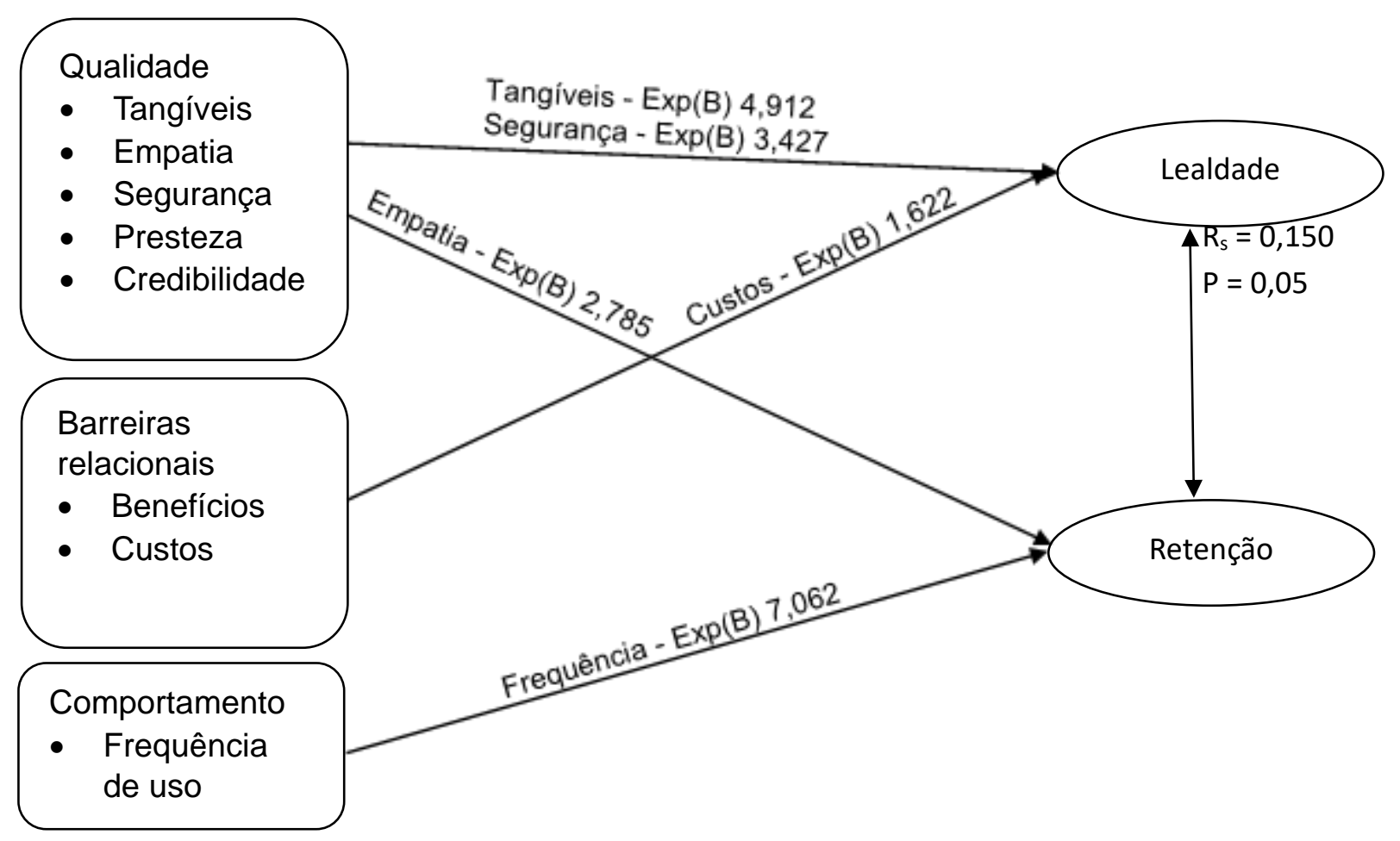

Fonte: Elaborado pelo autor

O quadro 4 apresenta um resumo dos principais achados dessa pesquisa 


\section{CONSIDERAÇÕES}

De fato, existe relação entre o comportamento de recompra e a lealdade na perspectiva atitudinal, entretanto, esta relação, para a amostra pesquisada, foi considerada baixa. Isto leva a crer que uma única estratégia não pode ser suficiente para manter níveis de retenção e de lealdade altos. Se a relação entre elas é baixa, é provável que ações diferentes sejam necessárias para produzir resultados específicos para retenção ou lealdade de clientes. Este resultado justifica a necessidade de se testar um modelo de antecedentes para cada uma das variáveis dependentes.

Com relação à qualidade, Pan et al. (2012) observaram que os preditores da lealdade comuns na literatura (incluindo qualidade percebida) se relacionam mais frequentemente com a lealdade na perspectiva atitudinal do que com 0 comportamento de retenção, afirmando que são escassos os estudos que utilizam alguma medida de comportamento associada à lealdade. Esta pesquisa evidenciou essa forte relação entre a percepção de qualidade do serviço e a lealdade na perspectiva atitudinal, porém, identificou uma relação limitada entre qualidade e retenção.

Conclui-se que a qualidade do serviço nas academias de ginástica pesquisadas é uma dimensão preponderante para o desenvolvimento da lealdade dos clientes. $O$ fator que mais se relaciona positivamente com a lealdade é 'tangíveis', seguido de 'segurança'.

Para o modelo preditivo da retenção, apenas a dimensão Empatia foi aceita. Conclui-se que os fatores relacionados com o bom atendimento e cortesia por parte dos funcionários podem levar a aumentos nos números da retenção de clientes em academias de ginastica. De uma forma geral, características como cordialidade e disposição para ajudar os clientes devem ser estimuladas e desenvolvidas por parte dos gestores de academias.

Com relação às barreiras à mudança, apenas os custos relacionais foram associados à lealdade, entretanto, não houve relação entre custos de mudança e retenção. Gwinner, Gremler e Bitner (1998) e Patterson e Smith (2003) afirmam que os benefícios sociais são ainda mais importantes nos serviços em que existe maior contato com o cliente, como serviços médicos, salão de beleza e serviços de fitness. Entretanto, esta afirmação não se mostrou verdadeira para a amostra deste estudo. 
Não houve relação significativa entre benefícios sociais e lealdade, nem também com a retenção. Assim, como descrito na literatura, os benefícios sociais, por si, não são determinantes para que haja a percepção dos custos de mudança (JONES et al., 2000). Embora eles sejam associados, são construtos independentes.

Neste sentido, conclui-se que as empresas devem investir em estratégias que levem ao aumento na percepção dos custos relacionais, a fim de aumentar significativamente os índices de lealdade de seus clientes. Considerando os benefícios financeiros advindos de uma carteira de clientes amplamente composta de clientes leais, não é concebível que existam funcionários no corpo de uma empresa, tratando seus clientes de maneira inapropriada, indiferentes às suas necessidades e, até mesmo, ignorando as atribuições de seu cargo.

Quanto à relação entre as características individuais e a retenção de clientes, conclui-se que quanto mais o cliente utiliza os serviços prestados pela academia, medido pela frequência de acessos, mais chances ele tem de renovar o contrato com a mesma. Isso pode estar intimamente ligado a um aumento na percepção do valor do serviço e, consequentemente, a um maior desejo de permanecer com um fornecedor.

Esta pesquisa contribui para o desenvolvimento do marketing de serviços no mercado do fitness e para a administração de uma maneira geral, trazendo numa mesma amostra, duas medidas diferentes associadas à lealdade (atitude e comportamento). Este aspecto permitiu que houvesse uma confrontação entre os diversos tipos de influência a que estão sujeitos os clientes de academias e duas consequências vitais para a sustentabilidade de um negócio: lealdade e retenção.

\section{REFERÊNCIAS}

AFTHINOS, Y.; THEODORAKIS, N. D.; NASSIS, P. Customer expectations of service in Greek fitness centers. Managing Service Quality, v. 15, n. 3, p. 245-258, 2005. https://doi.org/10.1108/09604520510597809

ARAÚJO, Carlos A. S. Identificação dos Fatores de Aderência em Programas de Atividade Física em Academias Utilizando Inteligência Computacional. 2010. 150 f. Dissertação (mestrado em engenharia biomédica) Programa de pós-graduação em engenharia biomédica. Universidade Federal do Rio de Janeiro, Rio de Janeiro, 2010. 
BROWN, T. J., CHURCHILL JR., G. A., PETER, J. P. Improving the measurement of service quality. Journal of Retailing, v. 69, n. 1, p.127, 1993. https://doi.org/10.1016/S00224359(05)80006-5

BURNHAM, Thomas A.; FRELS, Judy K.; MAHAJAN, Vijay. Consumer switching costs: a typology, antecedents, and consequences. Journal of the Academy of Marketing Science, v. 31, n. 2, p. 109-126, 2003. https://doi.org/.1177/0092070302250897

CARMAN, James M. Consumer perceptions of service quality: An assessment of the SERVQUAL dimensions. Journal of Retailing, v. 66, n. 1, p. 33, 1990.

CHELLADURAI, Packianathan; CHANG, Kyungro. Targets and standards of quality in sport services. Sport Management Review, v. 3, n. 1, p. 1-22, 2000.

https://doi.org/10.1016/S1441-3523(00)70077-5

CLEM, Theresa. Understanding Country Club Members' Loyalty: factors affecting membership renewal decisions. 2011. $81 \mathrm{f}$. Tese (doutorado) . Kent State University College and Graduate School of Education, Health, and Human Services, 2011.

COSTA, Ricardo J. G. Determinantes da Lealdade do Cliente no Health \& Fitness. 2011. 56 f. Dissertação (mestre em gestão do desporto). Universidade Técnica de Lisboa; Faculdade de Motricidade Humana, 2011.

CRONIN JR, J. Joseph; TAYLOR, Steven A. Measuring service quality: a reexamination and extension. The Journal of Marketing, p. 55-68, 1992. http://dx.doi.org/10.2307/1252296

CRONIN JR, J. Joseph; BRADY, Michael K.; HULT, G. Tomas M. Assessing the effects of quality, value, and customer satisfaction on consumer behavioral intentions in service environments. Journal of Retailing, v. 76, n. 2, p. 193-218, 2000.

https://doi.org/10.1016/S0022-4359(00)00028-2

CYARTO, Elizabeth V.; BROWN, Wendy J.; MARSHALL, Alison L. Retention, adherence and compliance: important considerations for home-and group-based resistance training programs for older adults. Journal of Science and Medicine in Sport, v. 9, n. 5, p. 402-412, 2006. https://doi.org/10.1016/j.jsams.2006.06.020

DICK, Alan S.; BASU, Kunal. Customer loyalty: toward an integrated conceptual framework. Journal of the Academy of Marketing Science, v. 22, n. 2, p. 99-113, 1994. https://doi.org//10.1177/0092070394222001

DRUCKER, Peter Ferdinand. O melhor de Peter Drucker: obra completa. São Paulo: Nobel, 2002.

ENTRIEL, Aparecida Laino. Uma proposta para o entendimento do comportamento do consumidor e gestão de marketing de serviços. Originalmente apresentado como tese de doutorado. Universidade Federal do Rio de Janeiro, 2008.

ERLICH, Paulo. Mentoria e adesão a atividade física: o caso dos usuários da academia CHESF. Originalmente apresentado como dissertação de mestrado. Faculdade Boa Viagem/DEVRY. Recife, 2010. 
FERRAND, Alain; ROBINSON, Leigh; VALETTE-FLORENCE, Pierre. The intention-torepurchase paradox: A case of the health and fitness industry. Journal of Sport

Management, v. 24, n. 1, p. 83-105, 2010. https://doi.org/10.1123/jsm.24.1.83

FERREIRA, Célia Marina Costa. Um estudo sobre fidelização e retenção de clientes na área do fitness. Originalmente apresentada como dissertação de mestrado. Instituto Politécnico de Castelo Branco; Escola Superior de Gestão, 2012.

FORNELL, C., Johnson, M. D., Anderson, E. W., Cha, J., \& Bryant, B. E. The American customer satisfaction index: nature, purpose, and findings. The Journal of Marketing, p. 718, 1996. http://dx.doi.org/10.2307/1251898

GARCIA, Fernández J.; SAÑUDO, Corrales B. Las experiencias de servicio en clientes mayores de 50 años y su influencia en la lealtad en centros de fitness privados.Kronos, v. 12, p. 74-83, 2013.

GIL, Antonio Carlos. Métodos e técnicas de pesquisa social. 6. ed. São Paulo: Atlas, 2008.

GONÇALVES, Celina R. N. Retenção de sócios no fitness: estudo do posicionamento, expectativas, bem-estar e satisfação. Originalmente apresentada como tese de doutorado. Universidade Técnica de Lisboa; Faculdade de Motricidade Humana, 2012.

GREMLER, Dwayne D.; GWINNER, Kevin P.; BROWN, Stephen W. Generating positive word-of-mouth communication through customer-employee relationships. International Journal of Service Industry Management, v. 12, n. 1, p. 44-59, 2001.

https://doi.org/10.1108/09564230110382763

GRÖNROOS, Christian. An applied service marketing theory. European Journal of Marketing, v. 16, n. 7, p. 30-41, 1982. https://doi.org/10.1108/EUM0000000004859

GUPTA, Sunil; ZEITHAML, Valarie. Customer metrics and their impact on financial performance. Marketing Science, v. 25, n. 6, p. 718-739, 2006.

https://doi.org/10.1287/mksc. 1060.0221

GWINNER, Kevin P.; GREMLER, Dwayne D.; BITNER, Mary Jo. Relational benefits in services industries: the customer's perspective. Journal of the Academy of Marketing Science, v. 26, n. 2, p. 101-114, 1998. https://doi.org/10.1177/0092070398262002

HAIR, Joseph F. et al. Análise multivariada de dados. Bookman, 2009.

HESKETT, James L. et al. Putting the service-profit chain to work. Harvard Business Review, v. 72, n. 2, p. 164-174, 1994.

JONES, Thomas O; SASSER, W. Earl. Why satisfied customers defect. Harvard Business Review, v. 73, n. 6, p. 88-\&, 1995. https://doi.org/10.1061/(ASCE)0742-

597X(1996)12:6(11.2)

JONES, Michael A.; MOTHERSBAUGH, David L.; BEATTY, Sharon E. Switching barriers and repurchase intentions in services. Journal of Retailing, v. 76, n. 2, p. 259-274, 2000. https://doi.org/10.1016/S0022-4359(00)00024-5 
JONES, Michael A.; MOTHERSBAUGH, David L.; BEATTY, Sharon E. Why customers stay: measuring the underlying dimensions of services switching costs and managing their differential strategic outcomes. Journal of Business Research, v. 55, n. 6, p. 441-450, 2002. https://doi.org/10.1016/S0148-2963(00)00168-5

JONES, Michael A.; REYNOLDS, Kristy E.;MOTHERSBAUGH, David L.; BEATTY, Sharon E. The positive and negative effects of switching costs on relational outcomes. Journal of Service Research, v. 9, n. 4, p. 335-355, 2007. https://doi.org/10.1177/1094670507299382

KIM, Daeshik; KIM, Susan Y. QUESC: an instrument for assessing the service quality of sport centers in Korea. Journal of Sport Management, v. 9, n. 2, p. 208-220, 1995. https://doi.org/10.1123/jsm.9.2.208

KOTLER, Philip; KELLER, Kevin Lane. Administração de Marketing. 12. ed. São Paulo: Pearson Prentice Hall, 2006.

LAM, Eddie T. C.; ZHANG, James J.; JENSEN, Barbara E. Service Quality Assessment Scale (SQAS): An instrument for evaluating service quality of health-fitness clubs. Measurement in Physical Education and Exercise Science, v. 9, n. 2, p. 79-111, 2005. https://doi.org/10.1207/s15327841mpee0902_2

LARSON, R.; FARBER, B. Estatística aplicada. 4. ed. São Paulo: Pearson Prentice Hall, 2010.

LEWIS, Michael. The influence of loyalty programs and short-term promotions on customer retention. Journal of Marketing Research, v. 41, n. 3, p. 281-292, 2004.

https://doi.org/10.1509/jmkr.41.3.281.35986

LOVELOCK, Christopher; WIRTZ, Jochen; HEMZO, Miguel Angelo. Marketing de serviços: pessoas, tecnologia e estratégia. São Paulo: Pearson Prentice Hall, 2011.

MADUREIRA, K.T.; GOSLING, M.; SOUKI, G. Q.; FILHO, C. G. Custos de mudança e seus impactos na falsa lealdade, nas emoções negativas e na comunicação boca-aboca na troca de operadoras de telefonia celular. Encontro da Associação Nacional de Pós-Graduação e Pesquisa em Administração. Rio de Janeiro, 2013.

MALHOTRA, Naresh K. Pesquisa de marketing: uma orientação aplicada. 4. ed. Porto Alegre: Bookman, 2006.

MCCARTHY, J. IHRSA's guide to membership retention: industry lessons on what and what not to do. Boston: IHRSA publications, 2004.

MORGAN, Robert M.; HUNT, Shelby D. The commitment-trust theory of relationship marketing. The Journal of Marketing, p. 20-38, 1994. http://sci-hub.tw/10.2307/1252308

NESLIN, Scott A.; GUPTA, S.; KAMAKURA, W., Lu, J.; MASON, C. H. Defection detection: Measuring and understanding the predictive accuracy of customer churn models. Journal of marketing research, v. 43, n. 2, p. 204-211, 2006. https://doi.org/10.1509/jmkr.43.2.204

NORTH, Adrian C.; HARGREAVES, David J.; MCKENDRICK, Jennifer. The influence of instore music on wine selections. Journal of Applied Psychology, v. 84, n. 2, p. 271, 1999. http://dx.doi.org/10.1037/0021-9010.84.2.271 
OLIVER, Richard L. Whence consumer loyalty?. The Journal of Marketing, p. 33-44, 1999. http://dx.doi.org/10.2307/1252099

OLIVER, Richard L. Satisfaction: a behavioral perspective on the customer. 2 ed. New York: M. E. Sharpe, 2010.

PAN, Yue; SHENG, Simon; XIE, Frank T. Antecedents of customer loyalty: An empirical synthesis and reexamination. Journal of Retailing and Consumer Services, v. 19, n. 1, p. 150-158, 2012. https://doi.org/10.1016/j.jretconser.2011.11.004

PARASURAMAN, Anantharanthan; ZEITHAML, Valarie A.; BERRY, Leonard L. A conceptual model of service quality and its implications for future research. The Journal of Marketing, p. 41-50, 1985. https://doi.org/10.2307/1251430

PARASURAMAN, A.; ZEITHAML, Valarie A.; BERRY, Leonard L. SERVQUAL: A multipleitem scale for measuring consumer perceptions of service quality. Journal of Retailing, $v$. 64, n. 1, p. 12-40, 1988.

PARASURAMAN, A.; BERRY, Leonard L BERRY, Leonard L.; ZEITHAML, Valarie A. Refinement and reassessment of the SERVQUAL Scale. Journal of Retailing, v. 67, n. 4, p. 420-450, 1991.

PARASURAMAN, A., ZEITHAML,V., BERRY, L. Reassessment of expectations as a comparison standard in measuring service quality: implications for further research. Journal of Marketing, v. 58, n. 3, p. 111-124, 1994a. http://doi.org/10.2307/1252255

PARASURAMAN, Arun; ZEITHAML, Valarie A.; BERRY, Leonard L. Alternative scales for measuring service quality: a comparative assessment based on psychometric and diagnostic criteria. Journal of Retailing, v. 70, n. 3, p. 201-230, 1994b. https://doi.org/10.1016/00224359(94)90033-7

PATTERSON, Paul G. Demographic correlates of loyalty in a service context. Journal of Services Marketing, v. 21, n. 2, p. 112-121, 2007.

https://doi.org/10.1108/08876040710737877

PATTERSON, Paul G.; SMITH, Tasman. A cross-cultural study of switching barriers and propensity to stay with service providers. Journal of Retailing, v. 79, n. 2, p. 107-120, 2003. https://doi.org/10.1016/S0022-4359(03)00009-5

PAYNE, A. Handbook of CRM: Achieving Excellence in Customer Relationship Management. Oxford: Butterworth-Heinemann Publications; Elsevier, 2006.

PECK, H.; Christopher, M.; Clark, M.; Payne, A. Relationship Marketing: strategy and implementation. Oxford: Butterworth-Heinemann Publications, 1999.

PEDRAGOSA, Vera; CORREIA, Abel. Expectations, satisfaction and loyalty in health and fitness clubs. International Journal of Sport Management and Marketing, v. 5, n. 4, p. 450-464, 2009. https://doi.org/10.1504/IJSMM.2009.023371

PEPPERS, Don; ROGERS, Martha. CRM Series marketing 1 to 1. 2ed. Makron Books, 2001. 
PETER, J. Paul; CHURCHILL JR, Gilbert A. Relationships among research design choices and psychometric properties of rating scales: a meta-analysis. Journal of Marketing Research, p. 1-10, 1986. https://doi.org/10.2307/3151771

PINE, B. Joseph; GILMORE, James H. Welcome to the experience economy. Harvard Business Review, v. 76, p. 97-105, 1998.

PORTER, Michael. Estrategia competitiva. Elsevier Brasil, 2004.

PORTO, Rafael Barreiros; SILVA, Jurema Barreto. Encadeamento comportamental que incentiva o tempo de contrato com clientes de academia de ginástica. Revista Brasileira de Marketing, v. 12, n. 4, p. 64-84, 2013. https://doi.org/10.5585/remark.v12i4.2554

PRODANOV, Cleber Cristiano; FREITAS, Ernani Cesar de. Metodologia do trabalho científico. 2. ed. Novo Hamburgo: Feevale, 2013.

REICHHELD, F. Loyalty rules! How today's leaders build lasting relationships. Boston: Harvard Business School Press, 2001.

REICHHELD, Frederick F. The one number you need to grow. Harvard Business Review, v. 81, n. 12, p. 46-55, 2003.

REICHHELD, Frederick; MARKEY, Rob Jr. Pergunta definitiva 2.0. Rio de Janeiro: Campus/Elsevier, 2012.

RIGBY, Darrell K.; LEDINGHAM, Dianne. CRM done right. Harvard Business Review, v. 82, n. 11, p. 118-130, 2004.

ROCHA, Viviane Moura; PONCHIO, Mateus Canniatti; FRANCISCO, Eduardo de Rezende. Lealdade do Consumidor e Programas de Fidelidade: uma análise topográfica do campo de conhecimento à luz da bibliometria, estatística espacial e redes sociais. Encontro da Associação Nacional de Pós-Graduação e Pesquisa em Administração. Rio de Janeiro, 2013.

ROJAS, Paola N. C. Aderência aos programas de exercícios físicos em academias de ginástica na cidade de Curitiba - PR. Originalmente apresentada como dissertação de mestrado. Universidade Federal de Santa Catarina, 2003.

SABÁ, Fabio. Aderência: a prática do exercício físico em academias. São Paulo: Manole, 2001.

SOUZA, J. C.; REIS, M. T. N.; MOURA, A. L. J. Identificando clientes propensos a encerrarem o relacionamento: um subsídio aos programas de relacionamento e à retenção de clientes no mercado bancário brasileiro. Encontro da Associação Nacional de Pós-Graduação e Pesquisa em Administração. São Paulo, 2009.

TAYLOR, Steven A.; BAKER, Thomas L. An assessment of the relationship between service quality and customer satisfaction in the formation of consumers' purchase intentions. Journal of Retailing, v. 70, n. 2, p. 163-178, 1994. https://doi.org/10.1016/00224359(94)90013-2 
VAVRA, Terry G. Marketing de Relacionamento - AfterMarketing: Como manter a fidelidade de clientes através do marketing de relacionamento. São Paulo: Atlas, 1993.

WHITE, Lesley; YANAMANDRAM, Venkat. Why customers stay: reasons and consequences of inertia in financial services. Managing Service Quality: An International Journal, v. 14, n. 2/3, p. 183-194, 2004. https://doi.org/10.1108/09604520410528608

YANG, Zhilin; PETERSON, Robin T. Customer perceived value, satisfaction, and loyalty: the role of switching costs. Psychology \& Marketing, v. 21, n. 10, p. 799-822, 2004.

https://doi.org/10.1002/mar.20030

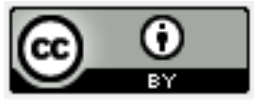

Artigo recebido em 30/01/2017 e aceito para publicação em 15/01/2018

DOI: http://dx.doi.org/10.14488/1676-1901.v18i1.2705 